\title{
Atypical Carcinoid Tumor
}

National Cancer Institute

\section{Source}

National Cancer Institute. Atypical Carcinoid Tumor. NCI Thesaurus. Code C72074.

A carcinoid tumor characterized by a high mitotic rate, often associated with the presence of necrosis and nuclear pleomorphism. 